\title{
Brain Natriuretic Peptide in The Diagnosis of Heart Failure
}

\section{IJCRR}

Section: Healthcare

Sci. Journal Impact

Factor: 6.1 (2018)

ICV: 90.90 (2018)

(c) (7) (3)

Copyright@IJCRR

\section{Balaji Tulse Dass ${ }^{1}$, R. Balamurugan²}

'Assistant Professor, General Medicine, Karuna Medical College, Vilayodi, Chittur, Palakkad, Kerala, India; 'Associate Professor, General Medicine, Karuna Medical College, Vilayodi, Chittur, Palakkad, Kerala, India.

\section{ABSTRACT}

Introduction: Heart failure (HF) is a global problem, with more than 20 million people affected. The overall prevalence of heart failure in developed countries is around $2 \%$. The significance of natriuretic peptides (NPs) is well established in the diagnosis of heart failure (HF).

Materials and Methods: In this cross-sectional study, 100 consecutive patients who presented with acute dyspnea to the Emergency department of a tertiary medical centre in Palakkad were included from December 2019-April $2020 . \quad$ The initial diagnosis for dyspnea was assigned to the emergency department. Later the patient was evaluated in the cardiology department and a final diagnosis was made. A rapid microparticle enzyme immunoassay (AXSYM SYSTEMS ABOTT) was used for the quantitative determination of BNP measurement. EDTA plasma of the patients was subjected to the estimation of BNP according to the manufacturer's instructions.

Results: Out of 100 patients, $79 \%$ had abnormal BNP levels (>100pg/ml); Out of these,6 In this study, among patients with elevated BNP levels $55 \%$ had abnormal ECG changes and $45 \%$ had normal ECG 1\% ( $=47$ ) were diagnosed to have $\operatorname{CCF}(p<0.01)$ and $39 \%$ had another diagnosis.

Conclusion: The results conclude that the measurement of BNP levels will help the clinicians to differentiate cardiac and noncardiac causes of dyspnea in the emergency setting.

Key Words: BNP, Dyspnea, Heart failure, ECG, Axsym Systems Abott

\section{INTRODUCTION}

Heart failure (HF) is a global problem with more than 20 million people affected worldwide. The overall prevalence of heart failure in developed countries is about $2 \%$. Heart failure prevalence rises with age and affects $6-10 \%$ of people over the age of $65 .^{1}$

Heart failure was once thought to arise primarily in the setting of a depressed left ventricular (LV) ejection fraction (EF). Several epidemiological research in this field has shown that approximately $50 \%$ of patients developing heart failure have a normal or preserved ejection fraction (EF $40-50 \%$ ). Accordingly, heart failure patients are now broadly categorized into one of two groups:

(1) HF with a depressed EF as(systolic failure) or

(2) HF with a preserved EF (diastolic failure). ${ }^{1}$

There is a significant challenge in the diagnosis of heart failure as there are no specific signs and symptoms of heart failure. The key for a correct and prompt diagnosis is to have a high index of suspicion, particularly for high-risk patients. The routine diagnostic aids include Electrocardiogram (ECG),2D ECHO, Doppler and Chest X-Ray.

The role of Natriuretic peptides (NPs) is well established in the diagnosis of heart failure (HF) with low levels of NPs being especially significant to exclude heart failure (HF). ${ }^{2}$

A meta-analysis published in 2015, studied the value of brain (B-type) NP (BNP), N-terminal proBNP (NT proBNP) and mid regional pro-atrial NP (MR-proANP) in the acute setting. It showed a high negative predictive value while using low cutoff levels (i.e. BNP $<100 \mathrm{pg} / \mathrm{ml}$; NT-proBNP $<300 \mathrm{pg} / \mathrm{ml}$, MR-proANP $<120 \mathrm{pmol} / \mathrm{l}){ }^{3}$

This study aimed to assess the role of BNP in the diagnosis of heart failure in patients presenting with acute breathlessness.

\section{Corresponding Author:}

Dr. R. Balamurugan, Department of Medicine, Karuna Medical College, Vilayodi, Chittur, Palakkad-678103, Kerala, India. Phone: 9842244881; E-mail: dr.balamur@gmail.com

ISSN: 2231-2196 (Print)

Received: 16.06 .2020
ISSN: 0975-5241 (Online)

Revised: 27.07 .2020
Accepted: 22.08 .2020
Published: 08.09.2020 


\section{MATERIALS AND METHODS}

In this cross-sectional study, 100 consecutive patients who presented with acute dyspnea to the Emergency department of Karuna Medical College, Palakkad were included.

Study period: December 2019-April 2020

Besides clinical examination, laboratory tests (renal function tests, $\mathrm{BNP}, \mathrm{Hb} \%$ ) and chest radiography, Echocardiography was done to all patients presenting with dyspnea.

Patients with left ventricular fractional shortening equal to or less than $25 \%$ were considered as having systolic dysfunction.

The initial diagnosis for dyspnea was assigned to the emergency department. Later the patient was evaluated in the cardiology department and a final diagnosis was made. Patients with both CCF and other concomitant diagnoses (such as sepsis leading to decompensated CCF) were considered as having CCF as the ultimate diagnosis because the study aimed to assess the ability of BNP in diagnosing CCF.

The study has been done by the ethical standards of the institutional ethical committee on human experimentation and with the Helsinki Declaration of 1975, as revised in 2013. The institutional ethical committee clearance has been obtained for the same. (No.KMC/IHEC/09/2019)

\section{BNP measurements}

A rapid microparticle enzyme immunoassay (AXSYM SYSTEM (ABOTT) was used for the quantitative determination of BNP measurement. Under aseptic conditions $3 \mathrm{ml}$ of blood was taken by venipuncture into an EDTA containing vacutainer and plasma was separated by centrifuging at 3000 $\mathrm{g}$ for 15 minutes. The EDTA plasma of the patients was further subjected to the estimation of BNP according to the AXSYM SYSTEMS operation manual.

\section{Test principle}

This test is a microparticle enzyme immunoassay forming antigen and antibody complexes coated microparticles If BNP present there is a complex formed with corresponding monoclonal antibody coated on the weels. The BNP values are calculated using AXSYM SYSTEM with standard calibrators.

A p-value of lesser than 0.05 was considered statistically significant. The data are reported as the mean $+/-\mathrm{SD}$ or the median, depending on their distribution.

\section{RESULTS}

In our study, $62 \%$ were males and $38 \%$ were females. $51 \%$ of the patients were in the age group between 40-60years; $36 \%$ were above 60 years and $13 \%$ were below 40 years. Among these, $7 \%$ in the age group of fewer than 40 years, $42 \%$ in the age group 40-60 years and $25 \%$ in the age group greater than 60 years had elevated BNP levels.[TABLE 1]

Out of 100 patients, $79 \%$ had abnormal BNP levels (>100pg/ $\mathrm{ml})$; Out of these, $61 \%(\mathrm{n}=47)$ were diagnosed to have $\mathrm{CCF}(\mathrm{p}<0.01)$ and $39 \%$ had other diagnosis.[CHART 1]

Among the study population, $62 \%$ were hypertensives, $63 \%$ were diabetics, $26 \%$ had renal failure, $43 \%$ were smokers. $58 \%$ were diagnosed with Coronary Artery Disease.

49 patients had abnormal chest X-Ray findings. The relation between these parameters and BNP are shown in [TABLE 2]

In this study, among patients with elevated BNP levels, 55 $\%$ had abnormal ECG changes and $45 \%$ had normal ECG. [CHART 2]

$41 \%$ of the patients with abnormal BNP had normal ejection fraction, $23 \%$ had mild ejection fraction, $20 \%$ had moderate ejection fraction and $16 \%$ had severe ejection fraction. [TABLE 3]

Among patient with elevated BNP levels, $56 \%$ had Akinesia / Dyskinesia and 23\% had normal regional wall motion. [CHART 3]

\section{DISCUSSION}

Natriuretic peptides are hormones of cardiac origin having natriuretic, diuretic, and vasodilatory effects ${ }^{4} \mathrm{BNP}$ belongs to a family of natriuretic peptides, which includes atrial natriuretic peptide (ANP) and C-type natriuretic peptide (CNP), with the latter mainly secreted by vascular endothelium. ANP and BNP are cardiac natriuretic peptides secreted by myocytes, with BNP mainly secreted by atrial myocytes in the normal heart. ${ }^{4}$ With an increase in cardiac wall stress, Brain natriuretic peptide (BNP), is secreted into the circulation. Thus, BNP is a robust biomarker to aid in differentiating cardiac and non-cardiac causes of breathlessness. ${ }^{5,6}$

The largest study using the Triage R POC in the Emergency room was the Breathing Not Properly trial enrolling 1586 patients presenting to the Emergency Room with acute dyspnea. 744 of the patients $(47 \%)$ were diagnosed to have heart failure based on clinical data. Mean BNP plasma concentrations at admission were 675 and $110 \mathrm{pg} / \mathrm{L}$ in patients with and without HF, respectively. ${ }^{7}$

The level of BNP was by itself the strongest predictor of a correct diagnosis of HF, with a cut-off of $100 \mathrm{pg} / \mathrm{L}$ (sensitivity $90 \%$, specificity $76 \%$ for distinguishing HF from other causes of dyspnea).

In our study, A B-type natriuretic peptide cut off value of $100 \mathrm{pg}$ per millilitre had a sensitivity of 97 percent, a specificity of 60 $\%$, and an accuracy of $82 \%$ for differentiating congestive heart 
failure from other causes of dyspnea. Lower values were associated with more accurate negative predictive values of about 94\%. This is consistent with the Breathing Not Properly trial. ${ }^{7}$

Among the patients with elevated BNP levels,61\% (N=47) were diagnosed to have $\mathrm{CCF}(\mathrm{p}<0.01)$. This is in comparison with a study done by Sheikh Ishaq, et al ${ }^{8}$ where $54 \%$ of CCF patients had elevated BNP levels.

Thus, in determining the correct diagnosis of heart failure, including BNP in the routine investigation along with clinical judgement would enhance the diagnostic accuracy.

\section{CONCLUSION}

Majority` of the patients presenting with Congestive Cardiac Failure fall in the age group of 40 to 60 years with males being affected more often than females. BNP levels can also be elevated in other condition like acute pulmonary embolism, renal failure, myocardial infarction and acute coronary syndrome. Using the cut off values of BNP of $100 \mathrm{pg} / \mathrm{ml}$, it can be concluded that low BNP levels are highly reliable in ruling out heart failure, whereas a value $>100 \mathrm{pg} / \mathrm{mL}$ is only a fair indicator of disease.

The results conclude that the measurement of BNP levels will help the clinicians to differentiate cardiac and non-cardiac causes of dyspnea in the emergency setting.

Acknowledgement: The authors acknowledge the immense help received from the scholars whose articles are cited and included in the references in this manuscript.

The authors acknowledge the medicine department staff for their cooperation in the study.

\section{Table 1: AGE VS BNP}

\begin{tabular}{lccc} 
AGE & \multicolumn{2}{c}{ BNP } & TOTAL \\
& NORMAL & ABNORMAL & \\
< 40 years & 7 & 6 & 13 \\
40 - 6o yrs & 9 & 42 & 51 \\
> 6o years & 5 & 31 & 36 \\
Total & 21 & 79 & 100 \\
$(\%)$ & $21 \%$ & $79 \%$ & $100 \%$ \\
\hline
\end{tabular}

BNP: Brain Natriuretic Peptide

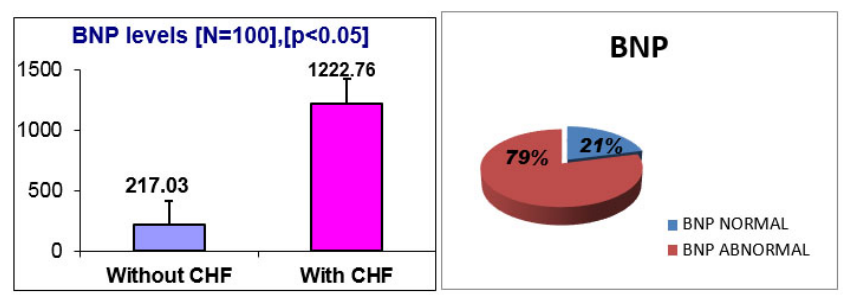

Chart 1: BNP.
The authors acknowledge the contribution of Dr.T.G.Ramya in drafting and editing the manuscript.

\section{Source of funding: NIL}

Conflict of interest: The authors report no conflict of interest in the work in this study.

\section{REFERENCES}

1. Mann DL, Chakinala M. Heart failure: Pathophysiology and Diagnosis In Kasper DL, Fauci AS, Hauser S, Longo D, Jameson J, eds.Harrison's Principles of Internal Medicine 19 ed.New York, NY: McGraw Hill;2015:1763.

2. Brunner-La Rocca H.P., Sanders-van Wijk S. Natriuretic peptides in chronic heart failure. Card. Fail. Rev. 2019;5:44-49.

3. Ponikowski P, Voors AA, Anker SD et al. 2016 ESC guidelines for the diagnosis and treatment of acute and chronic heart failure: the Task Force for the diagnosis and treatment of acute and chronic heart failure of the European Society of Cardiology (ESC) Developed with the special contribution of the Heart Failure Association (HFA) of the ESC. Eur Heart J. 2016;37:2129200.

4. Wang T.J. The natriuretic peptides and fat metabolism. $N$ Engl J Med.2012; 367:377-378.

5. Levin E.R., Gardner D.G., Samson W.K.Natriuretic peptides. N Engl J Med.1998; 339:321-328.

6. Cowie M.R., Jourdain P., Maisel A., et al. Clinical applications of B-type natriuretic peptide (BNP) testing. Eur Heart J.2003 24:1710-1718.

7. Maisel A, Krishnaswamy P, Nowak RM, McCord J, Hollander JE, Duc P, et al., for the Breathing Not Properly Multinational Study Investigators. Rapid measurement of B-type natriuretic peptide in the emergency diagnosis of heart failure. N Engl J Med 2002;347:161-7.

8. Ishaq, Sheikh, et al. "Brain natriuretic peptide (BNP): A diagnostic marker in congestive heart failure-induced acute dyspnea." International Journal of Medicine and Public Health, 2012; 2(4): $20-24$.

Table 2: Correlation between Various Parameters Tested with BNP

\begin{tabular}{lccc} 
PARAMETER & $\begin{array}{c}\text { ABNORMAL } \\
\text { BNP }\end{array}$ & $\begin{array}{c}\text { NORMAL } \\
\text { BNP }\end{array}$ & TOTAL \\
HYPERTENSION & 54 & 08 & 62 \\
DIABETES & 56 & 07 & 63 \\
RENAL FAILURE & 24 & 02 & 26 \\
SMOKING & 40 & 03 & 43 \\
CAD & 45 & 03 & 48 \\
ABNORMAL CXR & 41 & o8 & 49 \\
\hline
\end{tabular}

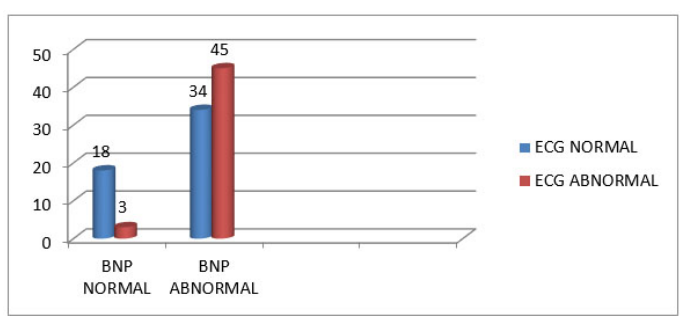

Chart 2: ECG VS BNP. 
Table 3: EF VS BNP

\begin{tabular}{lcccc}
\multicolumn{5}{c}{ BNP } \\
EF & Normal & Abnormal & Total & $(\%)$ \\
Normal & 13 & 18 & 31 & $52 \%$ \\
Mild & 2 & 10 & 12 & $20 \%$ \\
Moderate & 1 & 9 & 10 & $17 \%$ \\
Severe & 0 & 7 & 7 & $12 \%$ \\
Total & 16 & 44 & 60 & $100 \%$ \\
$(\%)$ & $27 \%$ & $73 \%$ & $100 \%$ & \\
\hline
\end{tabular}

EF: Ejection Fraction

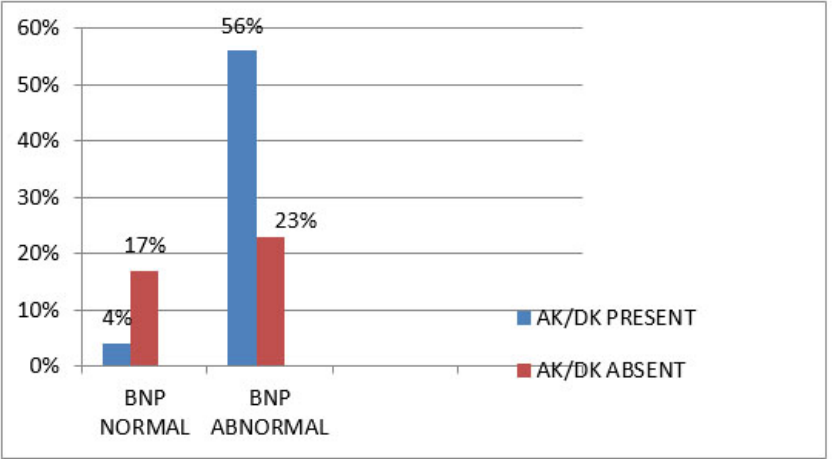

AK: Akinesia, DK: Dyskinesia

Chart 3: AK/DK VS BNP. 